\section{On the Evaluation of the Specific Mass Shift in the Ground States of Members of the He Isoelectronic Series}

\section{Subal Saha}

Department of Physics, Vivekananda College, Thakurpukur, West Bengal, India

and Sankar Sengupta

Department of Physics, Jadavpur University, Calcutta-32, West Bengal, India

Z. Naturforsch. 34a, 1538-1540 (1979); received August 20, 1979

A variation-perturbation method to calculate the specific mass shift in the ground state configuration of members of the He isoelectronic series is proposed. Through minimization of the second order energy, completely coupled and correlated orbitals are obtained in the Hartree Fock approximation. The influence of the Pauli principle and the electrostatic interaction on the motion of the electrons are automatically included while estimating the specific mass shift. This procedure also enables expectation values of the operator $\boldsymbol{r}_{1} \cdot \boldsymbol{r}_{2}$ to be calculated easily.

\section{Introduction}

The effect of the finite mass of the nucleus on an atomic Hamiltonian is two fold. Firstly, the reduced mass of the electron replaces its actual mass. Secondly, it induces a coupling between the linear momenta of the electrons. The coupling term is represented by $-(1 / M) \nabla_{1} \cdot \nabla_{2}$ in a.u. This is known as the specific mass shift operator, which plays an important role in the isotope shift of spectral lines. The term specific mass shift reflects the possibility of correlated motion of the electrons. In order to balance the correlated motion of the electrons there is movement of the nucleus and viceversa.

The contribution of the specific mass shift to the ground state energy is zero in the Hartree-Fock approximation for two-electron systems. But, as shown in this note, it is possible to evaluate the specific mass shift correction in the coupled Hartree Fock (CHF) approximation by extending the method developed by Sengupta and Mukherjee [1]. The operator $-(1 / M) \nabla_{1} \cdot \nabla_{2}$ operating on the ground state wave function of $\mathrm{He}$ and He-like ions

Reprint requests to $\mathrm{S}$. Chandra Saha, Vivekananda College, Department of Physics, Thakurpukur, Calcutta700063, West Bengal, Indien. gives rise to an angular function similar to the function of ${ }^{1} S$ symmetry of a $p^{2}$ configuration. The correction of the ground state wave function as a result of specific mass shift leads to correlated radial functions which improve the ground state energy considerably. While calculating the effect of the specific mass shift by the present procedure, the effects of the Pauli principle and the $l=1$ part of the classical expansion of electrostatic interaction on the movement of the electrons are automatically included. With the knowledge of the correlated wave functions obtained by the present method, one can easily calculate the values of the matrix elements of the operator $\boldsymbol{r}_{1} \cdot \boldsymbol{r}_{2}$. Expectation values of the operator $-(1 / M) \nabla_{1} \cdot \nabla_{2}$ and $\boldsymbol{r}_{1} \cdot \boldsymbol{r}_{2}$ are required in the investigation of the properties of atomic systems using oscillator strength sum rules, ef. [2] and [3].

\section{Theory}

The Hamiltonian $H$ of a two electron system (in a.u.) is :

$$
H=H_{0}+H^{\prime},
$$

where

$H_{0}=-\frac{1}{2}\left(\nabla_{1}^{2}+\nabla_{2}^{2}\right)-Z\left(\frac{1}{\boldsymbol{r}_{1}}+\frac{1}{\boldsymbol{r}_{2}}\right)+\frac{1}{\boldsymbol{r}_{12}}$

and

$$
H^{\prime}=-(1 / M) \nabla_{1} \cdot \nabla_{2} .
$$

$H^{\prime}$ is to be treated as perturbation to the zeroth order Hamiltonian $H_{0} . M$ denotes the nuclear mass. $\Psi_{0}$ and $E_{0}$ represent the unperturbed $H F$ wave function and energy, respectively. $H^{\prime}$ operating on $\Psi_{0}$ gives rise to a combination of spherical harmonics to be denoted $L$, where $L$ is given by

$$
L=\sum_{\alpha=-1}^{1}(-1)^{\alpha+1} Y_{1}^{\alpha}(1) Y_{1}^{-\alpha}(2) .
$$

Equation (2) represents the angular function of ${ }^{1} S$ symmetry arising from the (pp) configuration. Under the influence of mass polarization, the unperturbed single particle orbital $\psi_{\mathrm{K}}{ }^{0}$ is modified to $\varphi_{\mathrm{K}}$. Now, incorporating the perturbation concept, we represent any orbital as

$$
\varphi_{\mathbf{K}}=\frac{\psi_{\mathbf{K}}{ }^{0}+\delta \psi_{\mathbf{K}}}{\left[1+\left\langle\partial \psi_{\mathbf{K}} / \delta \psi_{\mathbf{K}}\right\rangle\right]^{1 / 2}}
$$


with

$$
\left\langle\psi_{\mathrm{K}} 0 / \delta \psi_{\mathrm{K}}\right\rangle=0 .
$$

Using these relations, the total wave function becomes

$$
\Phi=N\left[\Psi_{0}+\Psi_{1}+\Psi_{2}\right],
$$

where $N$ is the normalization constant and $\Psi_{1}$ represents determinants in which all rows contain $\psi_{\mathrm{K}}{ }^{0}$ type functions except one row in which $\delta \psi_{\mathrm{K}}$ occurs. Similarly in $\Psi_{2}$ two rows contain $\partial \psi_{\mathrm{K}}$ type functions. All these terms should not be retained since only a few of them can be connected to $\Psi_{0}$ through the perturbing Hamiltonian. The appropriate normalized total wave function thus reduces to

$$
\Phi=\left(1-\frac{1}{2}\left\langle\Psi_{2} / \Psi_{2}\right\rangle\right) \Psi_{0}+\Psi_{2} .
$$

Before applying the variational principal to minimize the energy with respect to the $\partial \psi$ 's we couple together two perturbed orbitals. This gives rise to interelectronic correlated functions and makes it possible to estimate the contribution of the mass polarization effect.

The total energy of the system can be expressed as

$$
E=\left\langle\Phi\left|H_{0}+H^{\prime}\right| \Phi\right\rangle=E_{0}+\Delta E .
$$

Using some properties of the HF equations for the unperturbed wave functions the expression for $\Delta E$ can be written in a simple form, keeping terms upto second order:

$$
\begin{aligned}
\Delta E= & -E_{0}\left\langle\Psi_{2} \mid \Psi_{2}\right\rangle+2\left\langle\Psi_{0}\left|H_{0}\right| \Psi_{2}\right\rangle \\
& +\left\langle\Psi_{2}\left|H_{0}\right| \Psi_{2}\right\rangle+\left\langle\Psi_{0}\left|H^{\prime}\right| \Psi_{2}\right\rangle \\
& +\left\langle\Psi_{2}\left|H^{\prime}\right| \Psi_{0}\right\rangle .
\end{aligned}
$$

While considering the effect of mass polarization the perturbation caused by electrostatic interactions, neglected in the $\mathrm{HF}$ approximation, is automatically included in the present calculation. This is manifested by the presence of first order terms in the perturbed energy expression.
$\Delta E$ is now minimized variationally. For this purpose, analytic expressions are chosen to represent $\partial \psi_{\mathrm{K}}$. The spin functions are taken to be of the same form as appearing in unperturbed orbitals. The angular functions are taken such that the symmetry demanded by $(2)$ is always satisfied. The radial function is assumed to be of the type

$$
\sum_{i} c_{\mathrm{K} i} r^{n_{k i}} e^{-\xi_{k i} r} \text {. }
$$

Suitable values of $n_{\mathrm{K} i}$ and $\xi_{\mathrm{K} i}$ are preassigned. Now it is evident that the variation parameters $C_{\mathbf{K} i}$ appear quadratically and biquadratically in $\Delta E$. We therefore define a new set of parameters $Q_{\mathrm{S}}=C_{\mathrm{K} i} C_{\mathrm{L} j}$. The biquadratic terms are now quadratic in the $Q$ 's and the quadratic terms are linear in the $Q$ 's. The energy minimization condition $\partial / \partial Q_{\mathrm{S}}(\Delta E)$ leads to a set of simultaneous linear equations in the $Q$ 's which can be solved by standard procedures. This reduces the independent variation of two sets of parameters to a variation of one set of parameters preserving the nature of the $\mathrm{HF}$ solution and leads to correlated functions. Further simplification of the energy expression is possible through symmetry properties when two electrons are equivalent.

\section{Results and Discussion}

Our corrections to the ground state energy and expectation values of $\boldsymbol{r}_{1} \cdot \boldsymbol{r}_{2}$ and $-(1 / M) \nabla_{1} \cdot \nabla_{2}$ for some $\mathrm{He}$ isoelectronic systems are condensed in Table 1. The unperturbed functions are taken from [4]. $\partial \psi$ is chosen to have eight parameters. Due to the symmetry of the problem the number of independent parameters reduces to only thirty six. Once

\begin{tabular}{|c|c|c|c|c|c|c|}
\hline \multirow[t]{2}{*}{ Atom or Ions } & \multicolumn{2}{|l|}{$\Delta E$ (a.u.) } & \multicolumn{2}{|c|}{$-(1 / M) \nabla_{1} \cdot \nabla_{2}\left(\mathrm{~cm}^{-1}\right)$} & \multicolumn{2}{|l|}{$\boldsymbol{r}_{1} \cdot \boldsymbol{r}_{2}$ (a.u.) } \\
\hline & Present & Ref. [5] & Present & Ref. [6] & Present & Ref. [3] \\
\hline${ }^{4} \mathrm{He}$ & $-0.2279-1$ & $-0.2169-1$ & $0.5878+1$ & $0.4785+1$ & $-0.7588-1$ & $-0.6237-1$ \\
\hline${ }^{7} \mathrm{Li}^{+}$ & $-0.2397-1$ & & $0.5622+1$ & $0.4960+1$ & $-0.1902-1$ & $-0.1799-1$ \\
\hline${ }^{9} \mathrm{Be}^{+}$ & $-0.2452-1$ & & $0.6155+1$ & $0.5619+1$ & $-0.7406-2$ & $-0.6858-2$ \\
\hline${ }^{11} \mathrm{~B}^{+}$ & $-0.2476-1$ & & $0.6470+1$ & $0.6046+1$ & $-0.3619-2$ & $-0.3416-2$ \\
\hline $12 \mathrm{C}^{+}$ & $-0.2491-1$ & & $0.7255+1$ & $0.6878+1$ & $-0.2031-2$ & $-0.1939-2$ \\
\hline
\end{tabular}
$\Delta E$ is minimized, it is straight forward to evaluate the quantities under consideration. The present results seem to support the conclusion of Taylor and Parr [5] that, for the ground state of two electron atoms, the angular correlation is more

Table 1. Values of Correction to the ground state energy, $-(1 / M) \nabla_{1} \cdot \nabla_{2}$ and $r_{1} \cdot r_{2}$ for the different members of the He isoelectronic sequence. The format $A \pm n$ means $A \times 10^{ \pm n}$. 
important than the radial one. Expectation values of the specific mass shift operator and $\boldsymbol{r}_{1} \cdot \boldsymbol{r}_{2}$ as obtained by Pekeris [6] and Dalgarno and Kingston [3] calculated with Hylleraas type of wave functions, are also listed in Table 1 . In view of the restricted form of the wave functions employed in

[1] S. Sengupta and A. Mukherjee, Phys. Rev. 166, 36 [1968].

[2] S. Saha and S. Sengupta, J. Quant. Spectrosc. Radiat. Transfer 20, 249 [1978].

[3] A. Dalgarno and A. Kingston, Proc. Roy. Soc. A, London 247, 245 [1958]. this work, the agreement between the different values is reasonably good. The agreement improves steadily with increasing values of $Z$. The method developed here is quite general and can be extended to larger systems without much labour.

[4] E. Clementi., Tables of Atomic Functions. IBM. J. Res. Suppl. 9, 2 [1965].

[5] G. R. Taylor, and R. G. Parr, Proc. Natl. Acad. Sci. 38, 154 [1952]

6] C. L. Pekeris, Phys. Rev. 112, 1649 [1950]. 